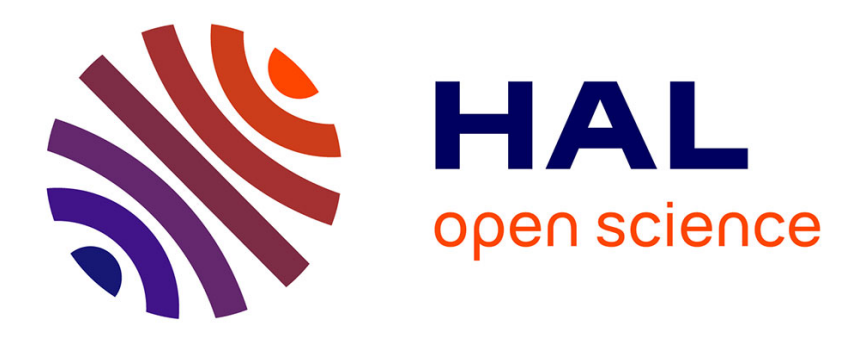

\title{
OPC model error study through mask and SEM measurement error
}

\author{
Mame Kouna Top, David Fuard, Vincent Farys, Yorick Trouiller, Patrick \\ Schiavone
}

\section{- To cite this version:}

Mame Kouna Top, David Fuard, Vincent Farys, Yorick Trouiller, Patrick Schiavone. OPC model error study through mask and SEM measurement error. Metrology, Inspection, and Process Control for Microlithography XXIV, Feb 2010, San Jose (CA), United States. in press. hal-00462229

\section{HAL Id: hal-00462229 \\ https://hal.science/hal-00462229}

Submitted on 8 Mar 2010

HAL is a multi-disciplinary open access archive for the deposit and dissemination of scientific research documents, whether they are published or not. The documents may come from teaching and research institutions in France or abroad, or from public or private research centers.
L'archive ouverte pluridisciplinaire HAL, est destinée au dépôt et à la diffusion de documents scientifiques de niveau recherche, publiés ou non, émanant des établissements d'enseignement et de recherche français ou étrangers, des laboratoires publics ou privés. 


\title{
OPC model error study through mask and SEM measurement error
}

\author{
Mame Kouna Top ${ }^{\mathrm{a}, \mathrm{b}}$, David Fuard ${ }^{\mathrm{b}}$ Vincent Farys $^{\mathrm{a}}$, Yorick Trouiller ${ }^{\mathrm{c}}$, Patrick Schiavone ${ }^{\mathrm{b}, \mathrm{d}}$ \\ ${ }^{\text {a }}$ STMicroelectronics 850 rue Jean Monnet, F 38926 Crolles Cedex, France \\ ${ }^{\mathrm{b}}$ LTM-CNRS, 17 Rue des Martyrs, 38054 Grenoble Cedex 9, France \\ ${ }^{\mathrm{c}}$ CEA-Leti, 17 Rue des Martyrs, 38054 Grenoble Cedex 9, France \\ ${ }^{\mathrm{d}}$ UMI 2958 CNRS-Georgia Tech, Georgia Institute of Technology, Atlanta GA 30332
}

\begin{abstract}
Mask and metrology errors such as SEM (Scanning Electron Microscopy) measurement errors are currently not accounted for when calibrating OPC models. Nevertheless, they can lead to erroneous model parameters therefore causing inaccuracies in the model prediction if these errors are of the same order of magnitude than targeted modeling accuracy. In this study, we used a dedicated design of hundreds of features exposed through a Focus Exposure Matrix (FEM). We measured the mask bias from target for these structures and investigated its impact on the model accuracy. For the metrology error, we compared the SEM measurements to AFM measurements for as much as 105 features exposed in various process conditions of dose and defocus. These data have then been used in a OPC model calibration procedure. We show that the impact of the metrology error is not negligible and demonstrate the importance of taking into account these errors in order to improve the reliability of the OPC models.
\end{abstract}

Keywords: Lithography, Modeling, OPC, Metrology, Mask, SEM, AFM.

\section{INTRODUCTION}

Optical Proximity Correction (OPC) is one of the key optical lithography enablers to improve the achievable resolution and pattern transfer fidelity in IC manufacturing. Immersion lithography scanners are now reaching the limits of optical resolution leading to more and more constraints on OPC for increased simulation fidelity [1]. In order to achieve the required accuracy OPC needs reliable input data for model calibration.

Unfortunately, OPC models often start on spurious basis: as an example, the mask writing errors are currently not taken into account and thus lead to errors in OPC modeling. Metrology errors such as CD-SEM (Critical Dimension Scanning Electron Microscopy) measurement errors also lead to wrong model parameters causing inaccuracies in the model prediction. The aim of this study is to determine how much the metrology error and mask bias error impact on the accuracy of Optical Proximity Correction (OPC) models.

In order to quantify the measurement noise impact on OPC models we compared two types of metrologies. The Scanning Electron Microscope (SEM) which is commonly used in the semiconductor industry because of its measurement speed is compared to the Atomic Force Microscope (AFM) which measurements are much slower but has as reputation to be very accurate [2]. On the other hand mask bias is a source of model error which gets detrimental to the latest technology node OPC models.

At first, we will describe our experimental conditions and then we will define the mask bias and the measurement noise we had. In the last section we will assess their impact on model reliability.

\section{EXPERIMENTAL CONDITIONS}

\subsection{Wafer characteristics}

We exposed two 300mm wafers on an ASML XT1700i 193nm immersion stepper using a dedicated mask targeting the $40 \mathrm{~nm}$ logic design rules. Wafers were analyzed after the photolithography step. 
The first wafers was exposed at nominal conditions of dose and defocus for every die of the full wafer, will be denoted "CDU" which stands for CD Uniformity. This wafer was used to compare the CD dispersion measured using different metrologies. In order to study the CD uniformity (CDU) we compared the SEM and AFM data dispersion using the following definition: $\mathrm{CDU}=3 \sigma$ where $\sigma$ is the standard deviation of the measured dimension over five chips. We used several types of structures. The features we used are $\mathrm{CD} / \mathrm{Space}$ matrix and Isolated Space features. For $\mathrm{CD} / \mathrm{Space}$ features, CDs range from 100 to $300 \mathrm{~nm}$ and space from $100 \mathrm{~nm}$ to infinite. Isolated Space range is from 80 to $100 \mathrm{~nm}$ and pad width range is from 90 to $900 \mathrm{~nm}$.

We exposed a second wafer (denoted "FEM") as a Focus Exposure Matrix with 28 conditions of defocus and dose. The defocus range is $0.04 \mathrm{um}$ and the dose change from $+/-0.4 \mathrm{~mJ}$ from nominal. The FEM wafer allows us to compare the model errors for different process conditions using the two metrology techniques. We also measured this wafer using AFM and SEM. This wafer contains five kinds of features. First we have dense line (on CD/Space matrix) feature where CDs are from 100 to $500 \mathrm{~nm}$ and space are from 70 to $300 \mathrm{~nm}$ or infinite for isolated lines. After that we have Isolated Space features where trench are from 100 to $500 \mathrm{~nm}$ and pad width are from 300 to $700 \mathrm{~nm}$. Line-end features are separate by 100 to $300 \mathrm{~nm}$. Finally, we have H features for which CD of measured H bar are from 50 to $150 \mathrm{~nm}$.

Dense line

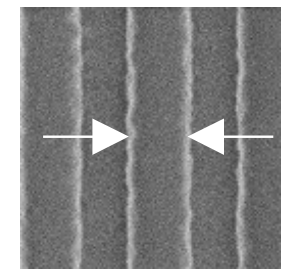

Isolated space

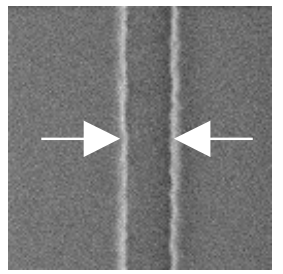

Line-end

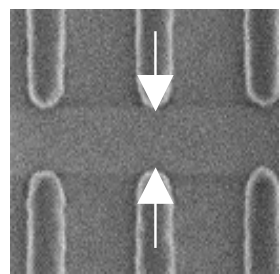

$\mathrm{H}$ feature

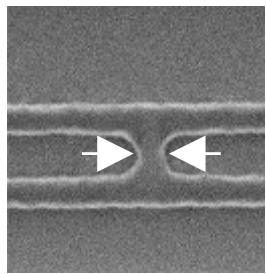

Figure 1: The different feature types used for the AFM-SEM measurement comparison

Figure 1 shows the different kind of features used on CDU and FEM wafer.. For FEM wafer also, model error is studied per kind of feature. We use different kinds of features in this study to identify the metrology error impact [3] and mask error impact on the model for each case.

\subsection{Metrology conditions}

The CD-SEM measurements were obtained using a Hitachi 9380 scanning electron microscope. The SEM measurements were performed using the same settings as the ones used on line for production control. Note that the focus adjustment is made outside the measurement area in order to avoid any shrinking of the resist during the focusing step. The CD-SEM is measured at a threshold of $80 \%$ of the image maximal intensity.

The AFM measurements were performed on the AFM-X3D from Veeco Instruments. AFM measurements were made very fast as on IC manufacturing conditions. For these measurements we scanned a length of $0.5 \mu \mathrm{m}$ with 10 scan lines. A 55nm wide flared tip was used. The AFM measurements are made at three different locations along the resist feature height.

- at the top of the resist (20nm from the top),

- at the middle of the resist

- and at the bottom of the resist (25nm from the bottom).

AFM profiles show that the top of the resist is rounded due to the optical lithography. This rounding is related to the feature edge slope which is itself correlated to the aerial image contrast. This assertion was confirmed with aerial image calculations. 


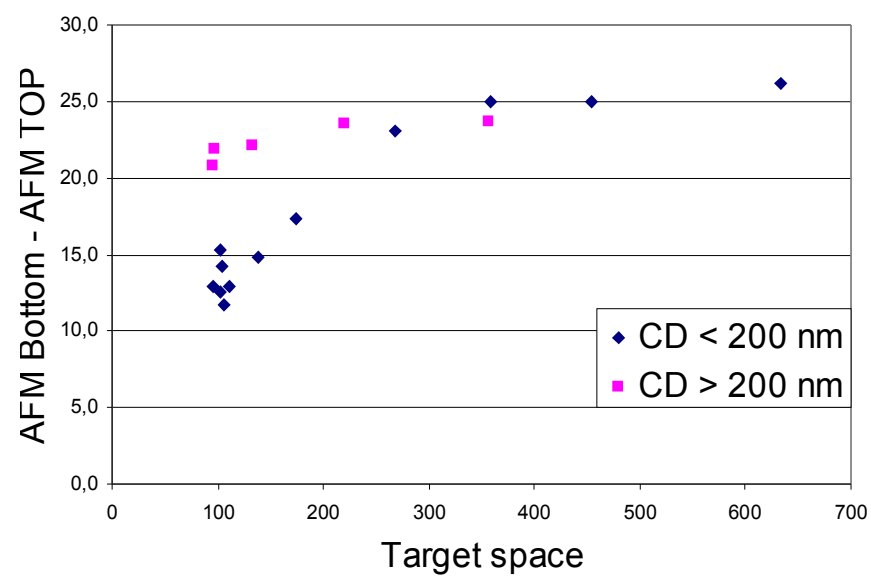

Figure 2 : the effects of corner rounding on the top of the resist for small features $(C D<200 \mathrm{~nm})$ in blue and large features $(\mathrm{CD}>200 \mathrm{~nm})$ in red

Figure 2 shows the difference between the AFM top and bottom measurements as a function of the nominal space width. We see that for features smaller than $200 \mathrm{~nm}$ (blue diamonds), the difference increases when the space decreases. The maximum difference can reach $15 \mathrm{~nm}$. For the larger features $(>200 \mathrm{~nm})$, we observe the same phenomenon but on a much smaller scale (less than $5 \mathrm{~nm}$ ).

The AFM measurement in the middle of resist does not suffer from corner rounding, but depends on the feature height, which vary from $95 \mathrm{~nm}$ to $106 \mathrm{~nm}$ for the whole features set.

This drawback does not occur for the AFM bottom measurement. It is always measured at the same height of $25 \mathrm{~nm}$ from the resist bottom. The bottom measurement is free from any deviation due to profile variation as well. We think that it can be considered as the most stable and reliable of these three different measurements along the features height.

In the following of this paper, otherwise specified, the AFM bottom measurement is used when AFM measurement are mentioned.

\section{MASK BIAS MEASUREMENT AND IMPACT OF BIAS MASK ON MODEL}

For the mask bias study we had the mask shop measure one third of all features of our test set. The results are shown on Figure 3.

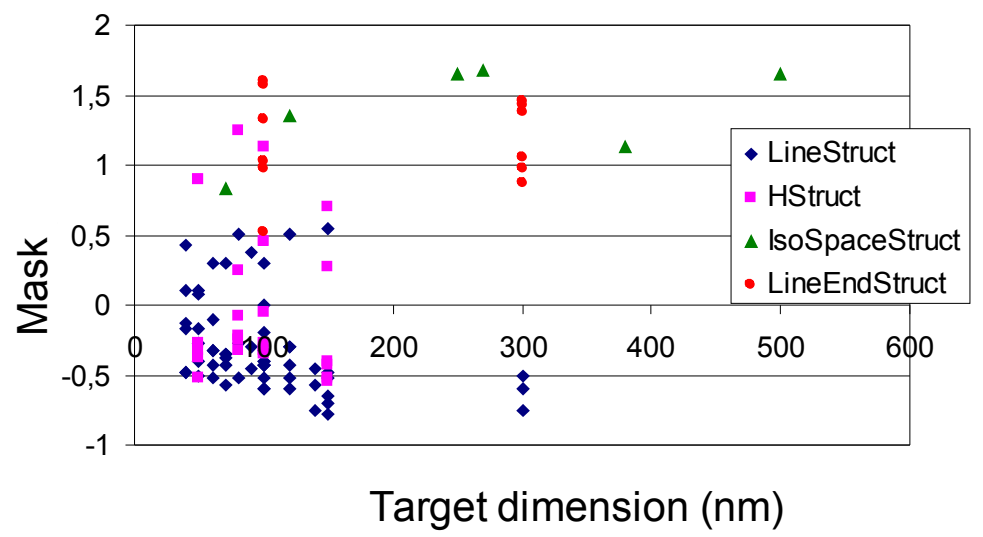

Figure 3 : Mask bias per feature type on FEM features

Figure 3 shows that the errors are small, with a maximum deviation to target of $2 \mathrm{~nm}$ at wafer scale. Line-end deviation is globally larger than for other features (positive offset $1.5 \mathrm{~nm}$ ). Lines show a slightly negative offset around $-0.5 \mathrm{~nm}$.

We built four models from the mask bias and FEM wafer data. The first two were built by fitting the measurement data to the OPC simulations using the nominal mask data (initial GDS). For the two others, (corrected GDS), we used the real 
mask data as measured by the maskshop instead. In each case, we built a constant threshold model (CTR) and a variable threshold model (VT5) model type from the Calibre software (Mentor Graphics). We used a subset made only from the smallest features (CDs from 40 to $100 \mathrm{~nm}$ and space from 50 to 100nm). We also tested the impact of mask bias on smaller features. All the results are gathered in Table 1.

\begin{tabular}{|l|l|l|l|}
\hline Features size & Model type & Initial GDS & Corrected GDS \\
\hline Large CD & RMS for CTR & 5.50 & 5.51 \\
\cline { 2 - 4 } & RMS for VT5 & 2.32 & 2.34 \\
\hline Small CD & RMS for CTR & 5.13 & 4.66 \\
\cline { 2 - 4 } & RMS for VT5 & 3,72 & 3,79 \\
\hline
\end{tabular}

Table 1: Comparison of mask bias correction model to the model creates with initial design for large and small CD size.

It can be seen that the mask bias does not have any impact in the case of large CDs whatever the model (CTR or VT5). There is a slight difference in RMS error for the small CDs with the threshold model, but it can hardly be considered as significant. The VT5 model which has a larger number of adjustable parameters completely masks the difference in mask size and provides the same residual error. This result shows that mask bias errors impact the model RMS of less of $1 \%$ for large CD and less of $10 \%$ for smaller CD in our case, when the mask bias error is of about $1 \%$ of the features size. This result also shows that the CTR model is about three time more sensitive to mask bias error than VT5 model in our case.

\section{COMPARISON OF AFM AND SEM METROLOGY}

In this section we will assess the metrology error by comparing AFM and SEM measurements. We will also study their impact on the OPC models. We will first use the CD Uniformity wafer to assess the correlation of measurement dispersion and CD Uniformity of the two methods. Then we will use the FEM wafer to study the impact of metrology noise on model.

\subsection{Measured CD dispersion}

We first compared the CD distribution measured using the 3D-AFM and the CD-SEM to check whether there are similarities in the dispersions.

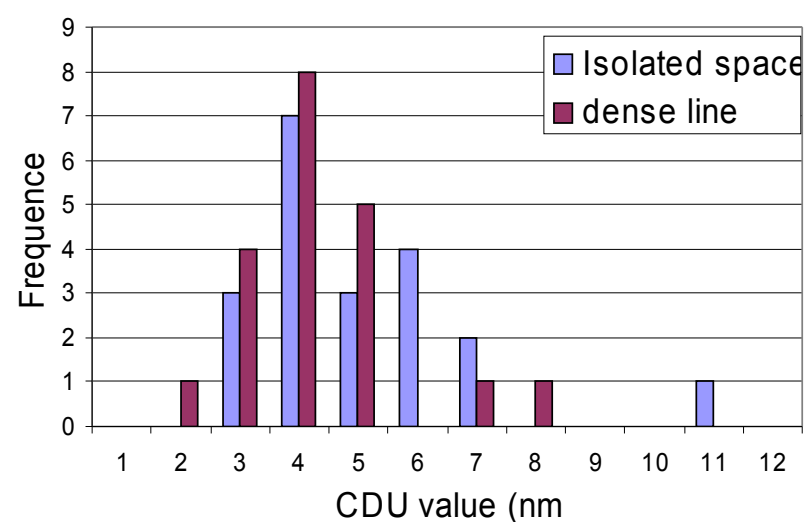

(a)

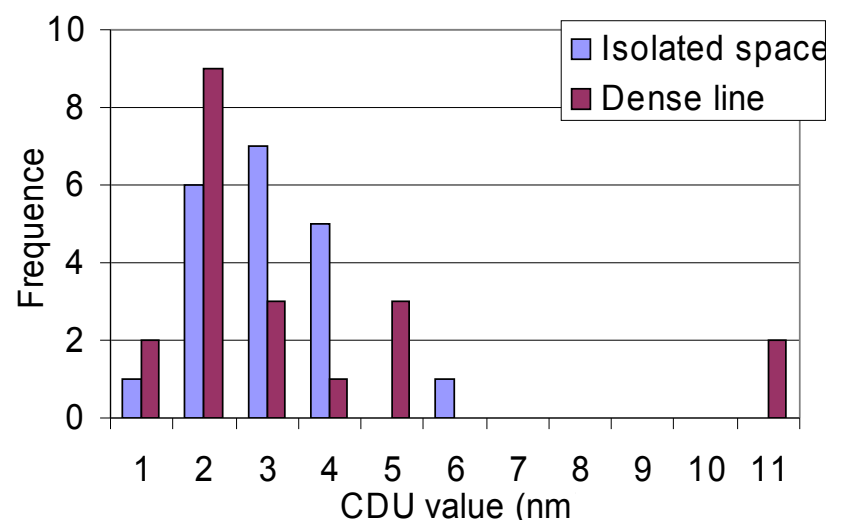

(b)

Figure 4: Histograms of AFM and SEM CD Uniformity measurements

Figure 4 shows the CD dispersion for the different kind of features. It can be seen from the next figure that the measures $\mathrm{CD}$ distributions are not similar for the lines as well as for the spaces. The mean deviation for the SEM metrology is $4.16 \mathrm{~nm}$ (standard deviation 1.63nm) for AFM metrology. And for SEM metrology, the mean deviation is of 4.63nm (standard deviation 2.17). This result shows that AFM CD dispersion through the five measured dies are as important as the dispersion of SEM measurement. 


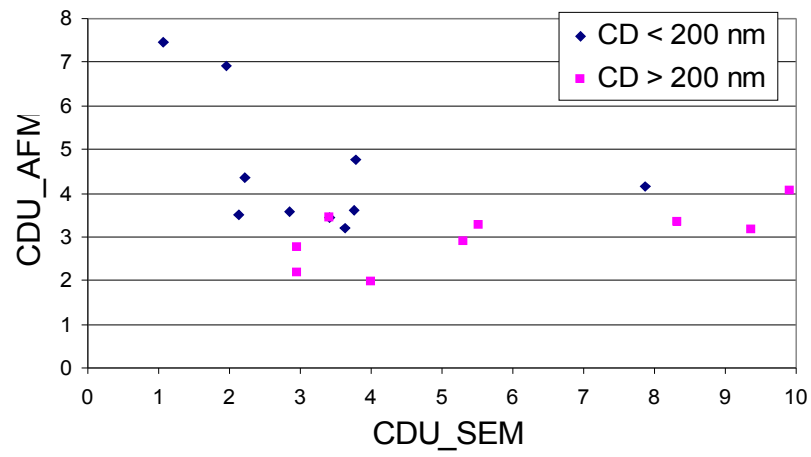

(a)

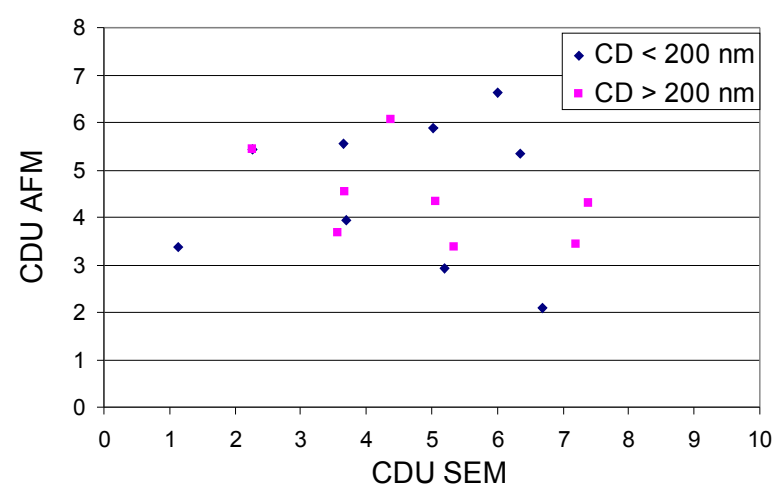

(b)

Figure 5: Histograms of AFM and SEM measurements CD Uniformity for active and poly layer

When investigating the AFM and SEM CD Uniformity similarity, Figure 5 shows that there is no difference either when we identify separately either small or large CDs. On the graphs CDs $<200 \mathrm{~nm}$ are plotted in blue and CDs $>200 \mathrm{~nm}$ are in pink. This figure illustrates the correlation of the AFM and SEM measurements within a very small range $(5+/-2$ nanometers around nominal). The plots hardly show any correlation between the measured values. In order to get a quantitative evaluation, we computed the correlation coefficients of the different datasets. The correlation coefficients are found to be very small ( 0.12 and 0.31 for lines and isolated spaces respectively), meaning no correlation between the two sets of data. This is no different for lines or spaces.

The absence of trend on these data simply means that there is no correlation between the dispersions of errors on AFM or SEM measurement at the scale of the wafer uniformity (few nanometers). Otherwise stated, the CD Uniformity is smaller or equal to the measurement noise of the AFM and SEM.

\subsection{FEM measurements for OPC model calibration:}

OPC models use calibration data from different conditions of dose and defocus. In this part of our study, we will compare the AFM and SEM calibration and try to assess their impact on model parameters.

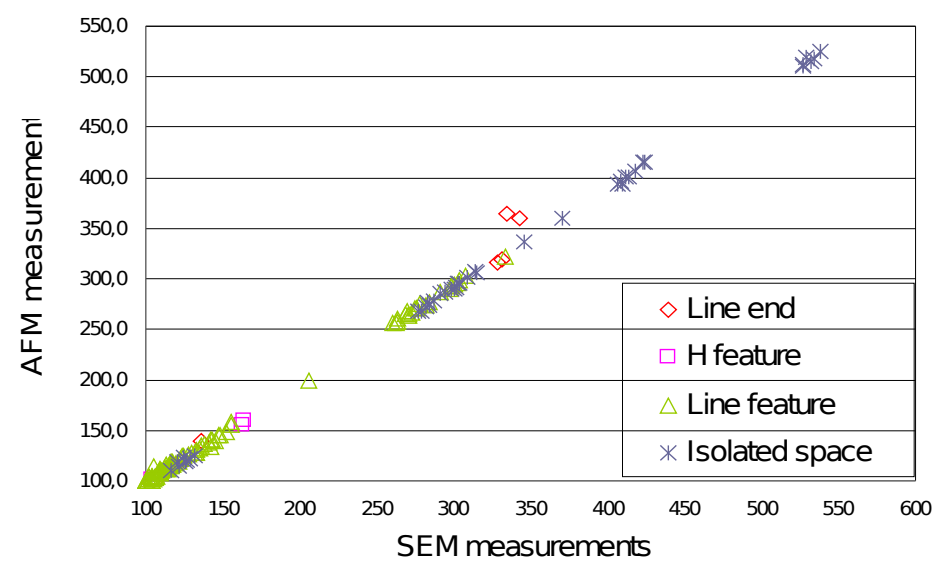

Figure 6: AFM measurement vs SEM measurement for FEM wafer' features

On Figure 6 as in Figure 5, the AFM measurements are plotted versus the SEM measurements. The different kinds of features are identified separately by different markers.

We can observe that all the points lie on the same regression line, except for the ends of lines. Contrary to what was observed in the previous section with uniformity measurements, we observe here a very good correlation between AFM and SEM measurements as shown in Table 3. 


\begin{tabular}{|l|l|l|l|l|}
\hline Feature type & Line end & H features & Lines & Spaces \\
\hline Correlation & 0,993 & 0.999 & 0.999 & 0.999 \\
\hline
\end{tabular}

Table 2 : Correlation of the AFM and SEM measurement for the different kind of features from the FEM data

There is not contradiction since here, the range of dimensions considered is much larger and overall much larger that the uncertainty of each metrology technique. Therefore their correlation is obviously almost perfect. The following table gives the averages and standard deviations of the AFM and SEM measurement differences.

\begin{tabular}{|l|l|l|l|l|}
\hline Features type & Line-end & H features & Lines & Spaces \\
\hline Mean $(\mathrm{nm})$ & $-1,56$ & 3,78 & 1,26 & 9,50 \\
\hline Standard deviation $(\mathrm{nm})$ & 15,22 & 2,40 & 2,45 & 3,53 \\
\hline
\end{tabular}

Table 3 : Mean and standard deviation of the AFM and SEM measurement difference

This table confirms the proximity between the AFM and SEM measurements for lines and H features with a difference of respectively 1 and $3 \mathrm{~nm}$ and a limited standard deviation of $2 \mathrm{~nm}$. However the measurement offset for space features is about 4-5 times larger, at 9nm, with a standard deviation of $3 \mathrm{~nm}$, which clearly shows a real measurement offset between AFM and SEM in this case. In the case of spaces, we found that the SEM recipe was poorly calibrated for this kind of measurement and this induces an offset against the AFM.

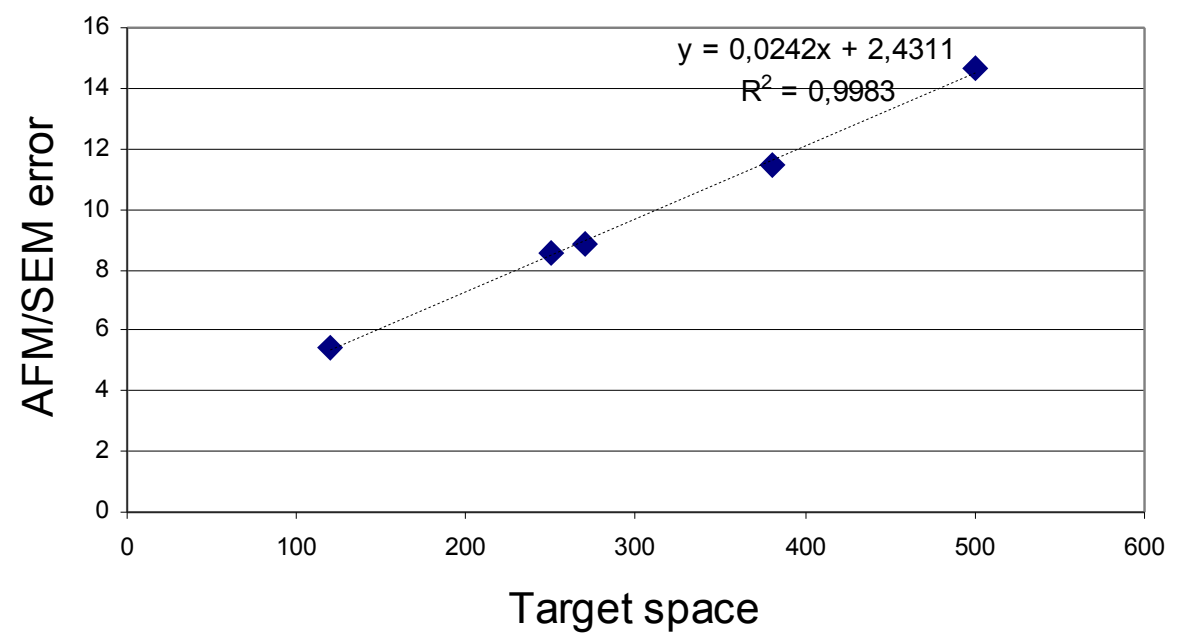

Figure 7: AFM/ SEM error for space feature depending to the GDS target space

Figure 7 shows this offset between AFM and SEM measurement depending on the target space in case of isolated space features. We found that this offset is highly correlated to the space width in the range 100-500 nm target space. Thus, this AFM-SEM offset as a function of the target space allows to greatly improve the SEM recipe.

We also note a different behavior for line ends, with a good proximity between the AFM and SEM measurements (1nm) but with about a five times larger standard deviation. For line-end features, the reason of the large dispersion of the AFM-SEM measurement bias is due to the AFM measurement. The AFM-X3D is not adapted to measure 2D structures in the substrate plane, which is typically the case for the line-ends. This translates to significantly increased measurement dispersion, even if the offset to the SEM is small.

\subsection{Impact of metrology on OPC models}

Using AFM or SEM measurements as calibration sets, we created two models. Our idea was to compare the model errors and check whether one of the two metrology techniques provides us a better modeling accuracy.

We created constant threshold models with the two datasets using the Calibre Workbench tool from Mentor Graphics. The same optical parameters were applied. The only parameter to be optimized is the aerial image (AI) threshold used for the CD calculation. The thresholds as well as the resulting RMS error are summarized in Table 4. 


\begin{tabular}{|l|l|l|}
\hline & AI threshold & RMS (nm) \\
\hline AFM dataset model & 0.1836 & 7.68 \\
\hline SEM dataset model & 0.1838 & 7.88 \\
\hline
\end{tabular}

Table 4 : AFM and SEM OPC model results

We note that the results are similar for both dataset in terms of RMS error and AI threshold. This is not surprising since the correlation between the two datasets showed to be almost perfect in the previous section.

These models were created using all structures at once. However, since we have a different number of items in each group of structure, the global model is weighted by the relative size of the groups. In the current case, the lines are by far the most numerous. We try to go further and fit the models feature by feature. Table 5 gives the RMS error obtained with the models calibrated from the AFM or SEM dataset feature by feature.

\begin{tabular}{|l|l|l|}
\hline Feature type & RMS for model from AFM data (nm) & RMS for model from SEM data (nm) \\
\hline Line & 8.6 & 8.5 \\
\hline H features & 5.4 & 5.9 \\
\hline Space & 4.3 & 5.4 \\
\hline
\end{tabular}

Table 5 : Results of the OPC model result by type of feature

This table shows that for $\mathrm{H}$ and line features there is not a great difference in terms of RMS whereas this does not hold for the other features. This result is in line with the results on AFM and SEM measurements presented in the previous section. The model extracted from the AFM measurements leads to a much larger residual error for line-ends. This is also consistent with the conclusion drawn from, that showed a poor quality of the measurement of 2D features with AFM.

For the space features, AFM measurements dataset permit to obtain better RMS than with SEM measurement. The reason explained in the previous section is due to the bad calibration of the SEM recipe for this particular type of features.

\section{CONCLUSION}

We compared two metrology techniques, namely AFM and SEM in order to assess the influence of the metrology on the OPC model reliability.

In terms of CD uniformity, the AFM and SEM are very comparable: we found that the AFM or SEM measurements variations around the nominal critical dimension are uncorrelated at the scale of less than $5 \mathrm{~nm}$. The dispersion around target (measurement noise) is of the same order than the lithography uniformity. This means that the CD Uniformity is less or equal to the measurement noise using AFM or SEM . The impact of the metrology on the OPC model quality extracted from full FEM data was overall found to be minor. We observed that our models were only impacted when one of the metrology candidates showed to be failing or known to be of poorer quality. This occurred for line-end (due to the capability of CD-AFM) and space features (due to the inappropriate settings of the SEM recipe)

\section{REFERENCES}

[1] Warren Grobman, Robert Boone, Cece Philbin, Bob Jarvis, "Reticule enhancement technology trends: resource and manufacturability implications for the implementation of physical designs", Proceedings of the 2001 international symposium on Physical design, Sonoma, California, United States, doi: 10.1145/369691.369730, p.45-51, (2001)

[2] J. Foucher, P. Faurie, A.-L. Foucher, V. Farys and M. Cordeau, "The measurement uncertainty challenge for the future technological nodes production and development", Proc. SPIE Vol. 7272, 72721K, (2009)

[3] T. Germer, "Modelling the effect of line profile variation on optical critical dimension metrology", Proc. SPIE 6518, 65180Z(2007).

\section{AKNOWLEDGEMENTs:}

The authors are grateful to Johan Foucher for his help with the AFM measurements 